\title{
Catechol-O-Methyltransferase Val158Met Modulation of Prefrontal-Parietal-Striatal Brain Systems during Arithmetic and Temporal Transformations in Working Memory
}

\author{
Hao-Yang Tan, ${ }^{1}$ Qiang Chen, ${ }^{1}$ Terry E. Goldberg, ${ }^{2}$ Venkata S. Mattay, ${ }^{1}$ Andreas Meyer-Lindenberg, ${ }^{1}$ \\ Daniel R. Weinberger, ${ }^{1}$ and Joseph H. Callicott ${ }^{1}$ \\ ${ }^{1}$ Clinical Brain Disorders Branch, Genes Cognition and Psychosis Program, National Institute of Mental Health, National Institutes of Health, Bethesda, \\ Maryland 20892, and 2Psychiatry Research Division, The Zucker Hillside Hospital, Glen Oaks, New York 11004
}

\begin{abstract}
Working memory (WM) is critically mediated by dopaminergic tuning of signal-to-noise in cortical neural assemblies. However, little is known about the distributed neuronal networks impacted by dopaminergic modulation in the component processes of WM. Here, we used the genotype of the Val158Met polymorphism in catechol-O-methyltransferase (COMT) as an index of relative cortical dopamine bioavailability and tuning efficiency, to examine the spatial and subprocess specificity by which dopaminergic modulation occurs within the prefrontal-parietal-striatal network during WM, thus empirically showing that dopamine plays key roles in updating and stabilizing new information at the neural systems level. In an event-related fMRI task dissociating component numerical WM subprocesses, baseline numerical size comparison engaged ventrolateral prefrontal cortical activation that correlated with COMT Val-allele load (COMT Val $>$ Met), while performing arithmetic transformations further engaged this genotype effect in dorsolateral prefrontal cortex (DLPFC), as well as in parietal and striatal regions. Critically, additional temporal integration of information in WM disproportionately engaged greater COMT Val $>$ Met effects only at DLPFC. COMT Val $>$ Met effects were also observed in DLPFC during encoding of new information into WM, but not at its subsequent retrieval. Thus, temporal updating operations, but less so the retrieval of already encoded representations, engaged relatively specific dopaminergic tuning at the DLPFC. Manipulating and rapidly updating representations were sensitive to dopaminergic modulation of neural signaling in a larger prefrontal-parietal-striatal network. These findings add to the integration of dopaminergic signaling in basic cortical assemblies with their roles in specific human brain networks during the orchestration of information processing in WM.
\end{abstract}

Key words: cerebral cortex; executive cognition; dopamine; fMRI; dopamine; genetics

\begin{abstract}
Introduction
Dopamine (DA) has been fundamentally implicated in the information processing characteristics of prefrontal cortex (PFC) neurons during working memory (WM) (Sawaguchi and GoldmanRakic, 1991; Seamans and Yang, 2004). Locally sustained firing of PFC neurons crucial in the maintenance of relevant information during the delay period of WM are stabilized against distracters through dopamine $\mathrm{D}_{1}$ receptors (Williams and Goldman-Rakic, 1995), which allow a focused augmentation of task-relevant signal-to-noise (Seamans et al., 2001). $\mathrm{D}_{2}$ receptor signaling might concurrently play critical roles in marking salience, prediction errors, and in rapidly updating and manipulating information through a network involving the PFC, posterior cortex, and
\end{abstract}

\footnotetext{
Received June 1, 2007; revised 0ct. 9, 2007; accepted 0ct. 12, 2007.

This work was supported by the United States National Institute of Mental Health Intramural Research Program and a National Institutes of Health fellowship (H.-Y.T.).

Correspondence should be addressed to either Dr. Hao-Yang Tan or Dr. Joseph H. Callicott, Unit for Dynamic Imaging Genetics, Clinical Brain Disorders Branch, National Institute of Mental Health, 10 Center Drive, Room 4C-

216, Bethesda, Maryland 20892. E-mail: tanh@mail.nih.gov or callicottj@mail.nih.gov. DOI:10.1523/JNEUROSCI.4041-07.2007

Copyright $\odot 2007$ Society for Neuroscience $\quad 0270-6474 / 07 / 2713393-09 \$ 15.00 / 0$
}

striatum (Goldman-Rakic, 1995; Mink, 1996; Tanaka et al., 2004; O’Reilly, 2006).

Less is known, however, about how these molecular and single-neuron properties translate spatially to the prefrontal-parietal-striatal network during various WM subprocesses in vivo. It has been conceptualized that the lateral PFC is hierarchically organized, where more dorsal and anterior prefrontal regions [e.g., dorsolateral PFC, DLPFC, Brodmann areas (BAs) 9, 10, and 46] were found to be engaged in higher-order processing such as in manipulating information or applying them in context, whereas the ventrolateral regions (e.g., VLPFC, BAs 44, 45 and 47) were engaged during simpler operations (Fuster, 1997; D'Esposito et al., 1999; Sakai and Passingham, 2002; Koechlin et al., 2003; Deco and Rolls, 2005). Because DA tuning of cortical neural assemblies is critical for their effective function in WM processes (Williams and Goldman-Rakic, 1995; Seamans and Yang, 2004; Vijayraghavan et al., 2007), it might be predicted that the hierarchically organized PFC functions would also be influenced by differential cortical DA bioavailability, with resultant effects on tuning efficiency in terms of regional activation (Egan et al., 2001b; Mattay et al., 2003) and functional integration (Meyer-Lindenberg et al., 2005; Winterer et al., 2006). Indeed, if 
DA is especially implicated in the updating and stabilization of representations (Seamans and Yang, 2004; Tanaka et al., 2004; O'Reilly, 2006), then executive WM tasks emphasizing encoding, manipulating, and temporally integrating information should be more dependent on changes in dopaminergic signaling than tasks emphasizing simple retrieval of already stabilized information. Some of the former processes are also likely to involve the DArich striatum, which has intimate connections to cortex in implementing the selective gating of information during rapid updating and manipulation in WM (Alexander et al., 1986; GoldmanRakic, 1995; Gruber et al., 2006; O'Reilly and Frank, 2006). For example, manipulating numerical representations might engage DA-dependent processes in the striatum, PFC, and numbersensitive regions in the parietal cortex (Dehaene et al., 2003; Hubbard et al., 2005). However, anterior regions in the DLPFC might be more specifically engaged during DA-dependent processing of higher-order temporal or episodic aspects of WM (Sakai and Passingham, 2002; Koechlin et al., 2003). Thus, we investigated if dopaminergic modulation integral to differing levels of WM processing could occur with a degree of spatial and process specificity within the human prefrontal-parietal-striatal network. We evaluated these predictions using candidate genetic variation in catechol-O-methyltransferase (COMT) known to influence cortical DA bioavailability (Chen et al., 2004).

\section{Materials and Methods}

Subjects. We initially studied 24 right-handed healthy subjects of European ancestry using event-related fMRI during a set of WM tasks based on computational and temporally varying information loads, in the context of candidate genetic variation in COMT (22q11.21; Mendelian Inheritance in Man 116790). Subjects were recruited from the National Institutes of Health Clinical Research Volunteer Program as part of the ongoing CBDB "Sibling Study" (Egan et al., 2001a). Subjects were all right-handed as assessed by the Edinburgh Handedness Inventory (Oldfield 1971) and were given a Structured Clinical Interview for the Diagnostic and Statistical Manual of Mental Disorders, fourth revision, to determine the presence of any psychiatric illnesses, a neurological examination, a battery of neuropsychological tests, an electroencephalogram (EEG), and a screening magnetic resonance imaging (MRI) examination. Exclusion criteria were inability to give informed consent, an intelligence quotient (IQ) $<70$, a history of substance abuse or psychiatric illness within the past 6 months, a history of significant neurological illness, and any focal abnormalities found by EEG or MRI. All subjects gave written consent before participation and were reimbursed for their time. The study was approved by the Institutional Review Board of the Intramural Program of the National Institute of Mental Health.

Genetic analysis. COMT Val158Met (rs4680) genotyping was obtained from venous blood using the Taqman $5^{\prime}$-exonuclease assay described previously (Chen et al., 2004). Genetic variation in COMT has become a popular approach through which differential dopaminergic modulation in the cortex can be investigated, as it is the major enzyme in DA catabolism within the cortex where the lack of DA transporters enhances the impact of this gene on DA levels (Karoum et al., 1994; Gogos et al., 1998; Chen et al., 2004; Tunbridge et al., 2006). In contrast, COMT does not impact on norepinephrine levels in prefrontal cortex (Gogos et al., 1998; Tunbridge et al., 2006). We examined a common polymorphism in the COMT gene, which results in a valine to methionine $\mathrm{Val}(108 / 158) \mathrm{Met}$ substitution, and gives rise to a significant reduction in its enzymatic activity in the cortex (Chen et al. 2004). Subjects were not preselected for COMT genotype. However, allele frequencies in this sample (Val at 0.54 and Met at 0.45 ) were similar to that in larger Caucasian populations and did not deviate from Hardy-Weinberg equilibrium (Fisher's exact test, $p>0.05)$. With relatively decreased synaptic DA as a function of COMT Val-allele load, we expected that cognitive processes sensitive to dopaminergic modulation would correspondingly engage a relatively increased neural response on fMRI in these individuals, representing a less efficient or detuned processing pattern (Egan et al., 2001b; Mattay et al., 2003; Bertolino et al., 2004; Meyer-Lindenberg et al., 2005, 2006). In other words, we used systems level "genetic imaging" of COMT val/met to serve as a proxy assay of dopaminergic modulation in WM subprocesses, and to generate in vivo information processing models of the distributed circuitry involved.

Cognitive paradigm. The event-related cognitive paradigm was performed in the MRI scanner after a brief training period $(\sim 5 \mathrm{~min})$. The paradigm allowed the separation of the encoding from the response phases in subsequent image analyses. This also potentially allowed for the isolation of cognitive processes of interest using planned contrasts of activations at the response phases. Various aspects of WM and cognitive control, along with a set of control tasks were included in the paradigm (see Fig. 1). Each set of WM or control tasks had in common a response phase, which required a sensorimotor response, numerical size judgment, or a more complex task that included numerical computation as well as numerical size judgment. Specifically, in the control tasks, the response phase lasted $3 \mathrm{~s}$, variants of which included the following: a motor task (M) whereby subjects pressed either the right or left response button based on an instruction projected on the screen; a numerical size judgment task $(\mathrm{J})$ in which subjects chose the number on the right or left based on an instruction to choose either the larger or the smaller number; and a numerical computation and size judgment task (CJ) in which subjects performed a numerical subtraction of 2 or 3 from either the left or right number, and made a numerical size judgment as instructed. In the WM tasks, 2 numbers were first displayed over $0.5 \mathrm{~s}$ for encoding (E). This was followed by a $4 \mathrm{~s}$ maintenance interval during which the screen was blank, and the subsequent $3 \mathrm{~s}$ response phase. In the WM retrieval and numerical size judgment task (E_RJ), subjects then pressed the left or right button based on an instruction to choose the larger or smaller remembered number. In the numerical computation and size judgment in WM task (E_CJ), subjects performed a numerical subtraction of 2 or 3 from the remembered number on the left or right as indicated, and made a numerical size judgment as instructed. In each trial, all the numbers were single digits from 0 to 9 ; the two numbers on which the numerical size judgment was ultimately performed (after numerical computation if applicable) were equally balanced across 0 to 9 , and equally likely to differ by either 1 or 3 units; numerical computation was equally likely on the left or right number, with correct responses equally balanced on the left or right, and equally likely to be the larger or smaller number. A jittered rest interval lasting $4 \mathrm{~s}$ to $8.5 \mathrm{~s}$ followed each trial. In each $\sim 11 \mathrm{~min}$ run, 10 trials of each task were performed in an order that was optimized using a sequencing program (Wager and Nichols 2003). Each subject performed two runs of the cognitive paradigm in the scanner.

Analysis of behavioral and demographic data. Behavioral performance on tasks was analyzed as two by two factorials using ANOVA separately for accuracy and reaction time. The first factor described whether tasks engaged computation whereas the second factor described whether tasks engaged a WM maintenance interval. We subsequently used one-way ANOVAs to examine for any COMT genotype effect on age, education, $\mathrm{IQ}$, and task performance accuracy and reaction time. Statistical significance was set at $p<0.05$.

MRI protocol. Whole brain blood oxygen level-dependent functional MRI (fMRI) data were collected on a 3-T scanner (General Electric, Milwaukee, WI) with a General Electric echo-planar imaging pulse sequence acquisition of 24 contiguous slices (echo time, $30 \mathrm{~ms}$; repetition time, $2 \mathrm{~s}$; flip angle, $90^{\circ}$; field of view, $24 \mathrm{~cm}$; matrix, $64 \times 64$; voxel dimensions, $3.75 \times 3.75 \times 5 \mathrm{~mm}$ ). The first four scans were discarded to allow for signal saturation. Stimuli were presented via a back-projection system, and responses were recorded through a fiberoptic response box, which allowed the measurement of the accuracy and reaction time for each trial.

Analysis of imaging data. The fMRI data were preprocessed and analyzed with SPM2 software (Wellcome Department of Cognitive Neurology, London, UK; http://www.fil.ion.ucl.ac.uk/spm) implemented in Matlab (Mathworks, Natick, MA). Images for each subject were slice timing corrected, realigned to the first volume in the time series, and corrected for head motion. Images were then spatially normalized into standard stereotaxic space (Montreal Neurologic Institute template) us- 


\section{Encoding phase}

Response phase
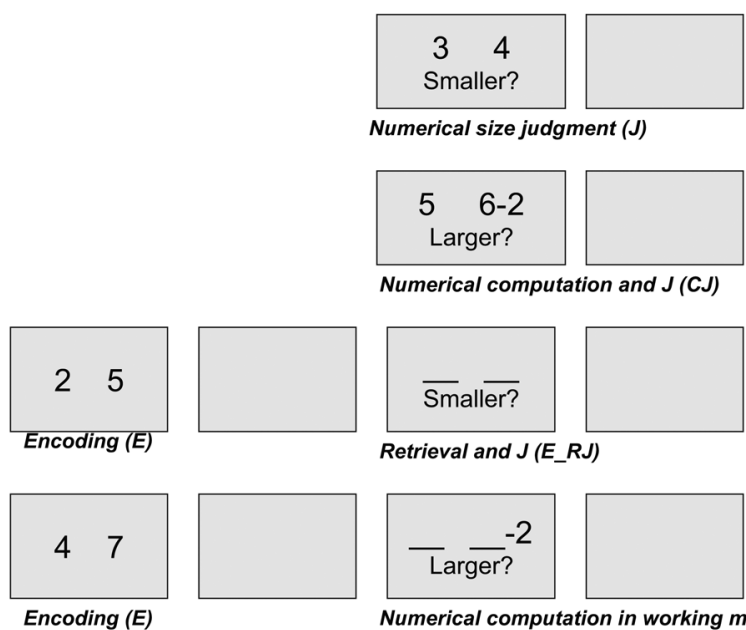

Retrieval and J (E_RJ)
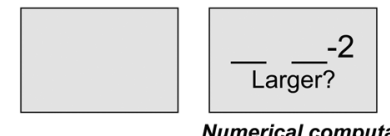
Nu

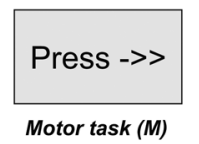

$0.5 \mathrm{~s}$ 3s
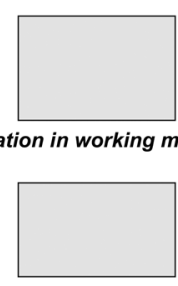

Rest: $4-8.5 \mathrm{~s}$

Figure 1. The event-related numerical WM task paradigm.

ing a 12-parameter affine model, and resampled to voxel size $2 \times 2 \times 2$ $\mathrm{mm}$. Spatial smoothing was applied with a Gaussian filter set at $8 \mathrm{~mm}$ full-width at half-maximum. After realignment, datasets were individually examined for motion as demonstrated by a small motion correction ( $<2 \mathrm{~mm}$ translation, $<1.5^{\circ}$ rotation). Two subjects were excluded for excessive movement.

fMRI responses were modeled using the canonical hemodynamic response function, ratio normalized to the whole-brain global mean to control for systematic differences in global activity, and temporally filtered using a high-pass filter of $128 \mathrm{~s}$. Events were modeled for correctly performed trials during each task phase at M, J, CJ, E, E_RJ, and E_CJ (Fig. 1). Incorrect responses and residual movement parameters were also modeled as regressors of no interest. Planned contrasts reflecting the following putative cognitive processes were performed: motor response ( $\mathrm{M}>$ baseline); numerical size judgment $(\mathrm{J}>\mathrm{M}$; subtracting $\mathrm{M}$ putatively removed the sensorimotor task components, including visual components of the instructional text, e.g., "larger," "smaller," or "press"); numerical computation outside WM $(\mathrm{CJ}>\mathrm{J})$; encoding into WM $(\mathrm{E}>$ baseline); retrieval from WM (E_RJ $>\mathrm{M})$; numerical computation in $\mathrm{WM}$ and its retrieval $\left(\mathrm{E} \_\mathrm{CJ}>\mathrm{J}\right.$ ); numerical computation in WM (E_CJ > E_RJ; this contrast potentially isolated mentally integrating probe, e.g., "-2," and remembered numbers, as well as computing or rapidly updating the relevant number, over and above simple retrieval and motor responses); and finally, the temporal integration of encoded information and probe information in WM (E_CJ > CJ; this contrast reflects manipulation of information in WM versus simple manipulation, or temporal integration of probe information with the encoded numbers, above and beyond simple computation/manipulation).

These contrasts were subsequently taken to a second-level group analysis in which intersubject variability was treated as a random effect. In evaluating the main effect of each of the above putative cognitive processes, a threshold of $p<0.05$ corrected for false discovery rate (FDR) (Genovese et al. 2002) within the whole brain search volume was applied. As for the COMT Val158Met genotype effects in each of the cognitive contrasts, we searched within functional regions of interest (ROIs) defined as those areas with significantly activated main effects of task that were located in the prefrontal cortex, posterior parietal cortex or striatum, in accordance with our previous hypotheses about these WM net- work regions. Within these ROIs, we regressed the COMT Val158Met genotype effects (Val/ $\mathrm{Val}>\mathrm{Val} / \mathrm{Met}>\mathrm{Met} / \mathrm{Met}$, abbreviated as COMT $\mathrm{Val}>\mathrm{Met}$ ) to identify voxels in which BOLD activation correlated with COMTVal allele load, putatively reflecting regions modulated by decreasing synaptic DA bioavailability and reduced tuning efficiency (Egan et al., 2001b; Mattay et al., 2003; Meyer-Lindenberg et al., 2005, 2006; Bertolino et al., 2006). These effects were thresholded at $p<0.05$, corrected for the ROI volume using Gaussian Random Field theory (Worsley et al. 1996). Voxels in the ROIs with COMT effects at a more lenient $p<$ 0.01 uncorrected were also reported.

Finally, we investigated functional integration between striatum and cortex in the context of the E_CJ task phase and COMT genotype to examine the assumption that increased activation in relation to COMT Val allele load reflected poorer neural tuning and reduced functional coupling within this WM network. This would be predicted by electrophysiological and imaging findings of decreased signal coherence within the WM network in states of suboptimally reduced cortical dopamine (Spencer et al., 2004; Meyer-Lindenberg et al., 2005; Winterer et al., 2006). Specifically, we examined psychophysiological interaction (PPI) as implemented in SPM2 (Friston et al., 1997), and measured how activity in the striatum during the E_CJ task phase covaried with that in prefrontal and parietal cortex. The striatum was chosen as the seed region because of its established functional and structural connectivity to extensive regions in cortex (Alexander et al., 1986). The seed was defined by sphere of $10 \mathrm{~mm}$ radius centered at a left caudate activation peak during E_CJ versus rest $(-1624-10 ; t=5.04 ; p<0.05$ FDR, corrected $)$. This area also overlapped with the peak in the E_CJ versus E_RJ contrast where COMT Val>Met effects were found (see Results). However, note that we chose the seed ROI from the E_CJ main effect, independent of the COMT effects. Hence, this analysis was orthogonal to the COMT activation findings, providing another dimension by which the activation data could be interpreted. A general linear model was then constructed at the first-level using three regressors: (1) the deconvolved bold signal from the caudate seed region, (2) the E_CJ task-related activation onsets, and (3) the interaction term between the first and the second regressor. Contrasts for this interaction term corresponds to brain regions considered to vary together as a functional network with the caudate ROI. These contrasts were then taken to a second-level random-effects analysis to examine prefrontal and parietal cortical regions being thus engaged by striatal and task-related functional coupling that also varied with cortical DA reflected by COMT Met allele load (i.e., Met-Met $>$ Val-Met $>$ Val-Val). The cortical regions examined were constrained within prefrontal and parietal activation masks, defined as areas significantly activated in E_CJ versus rest at $p<0.05$ (FDR corrected). The PPI analyses were thresholded at $p<0.01$ uncorrected, given the focused search for hypothesized higher-level COMT-related changes in WM network functional integration to be interpreted alongside effects of COMT on activation.

\section{Results \\ Demographic and behavioral results}

Tasks requiring computation (CJ and E_CJ) (Table 1) were reliably associated with lower accuracy $\left(F_{(1,21)}=33.75 ; p<0.001\right.$ for the main effect of computation) (supplemental Fig. 1, available at www.jneurosci.org as supplemental material) and slower reaction time $\left(F_{(1,21)}=477 ; p<0.001\right.$ for the main effect of computation) (supplemental Fig. 2, available at www.jneurosci.org as 
Table 1. Behavioral and demographic findings

\begin{tabular}{|c|c|c|c|c|c|c|c|c|}
\hline & VV & SD & VM & SD & MM & SD & $F$ & $p$ \\
\hline & $(n=8)$ & & $(n=8)$ & & $(n=6)$ & & & \\
\hline Age & 28.17 & 7.11 & 33.38 & 7.09 & 27.00 & 9.62 & 1.78 & 0.19 \\
\hline Gender (number of males) & 4 & & 5 & & 4 & & $\chi^{2}=0.45$ & 0.80 \\
\hline Education & 16.17 & 2.71 & 16.75 & 2.60 & 16.80 & 3.27 & 0.09 & 0.91 \\
\hline WAIS IQ & 101.0 & 13.6 & 109.4 & 14.1 & 103.8 & 7.2 & 0.81 & 0.46 \\
\hline \multicolumn{9}{|l|}{ Accuracy } \\
\hline Numerical size judgement (J) & 0.908 & 0.074 & 0.900 & 0.104 & 0.930 & 0.027 & 0.37 & 0.69 \\
\hline Numerical computation and J (CJ) & 0.867 & 0.113 & 0.756 & 0.247 & 0.850 & 0.141 & 1.05 & 0.37 \\
\hline Retrieval and J (E_RJ) & 0.933 & 0.061 & 0.969 & 0.053 & 0.920 & 0.076 & 0.12 & 0.89 \\
\hline \multicolumn{9}{|c|}{ Numerical computation in working memory } \\
\hline and $\mathrm{J}\left(\mathrm{E} \_\mathrm{CJ}\right)$ & 0.733 & 0.061 & 0.700 & 0.235 & 0.770 & 0.110 & 0.35 & 0.71 \\
\hline Motor task (M) & 0.992 & 0.020 & 0.969 & 0.059 & 0.980 & 0.045 & 0.28 & 0.76 \\
\hline \multicolumn{9}{|l|}{ Reaction time (s) } \\
\hline Numerical size judgement (J) & 1.388 & 0.289 & 1.407 & 0.263 & 1.318 & 0.148 & 0.65 & 0.53 \\
\hline Numerical computation and J (C) & 1.983 & 0.256 & 1.795 & 0.376 & 1.908 & 0.113 & 0.73 & 0.49 \\
\hline Retrieval and J (E_RJ) & 1.152 & 0.191 & 1.091 & 0.204 & 1.130 & 0.156 & 0.29 & 0.75 \\
\hline \multicolumn{9}{|c|}{ Numerical computation in working memory } \\
\hline and $J\left(E \_C\right)$ & 1.804 & 0.256 & 1.849 & 0.204 & 2.041 & 0.229 & 0.24 & 0.79 \\
\hline Motor task (M) & 0.918 & 0.206 & 0.918 & 0.110 & 0.911 & 0.117 & 0.17 & 0.85 \\
\hline
\end{tabular}

There were no significant demographic or performance differences across COMT genotype groups. VV, Val-homozygote; VM, Val-Met hetrozygote; MM, Met-homozygote.

supplemental material) relative to the tasks without calculation $(\mathrm{J}$ and E_RJ). Tasks requiring WM maintenance (E_RJ and E_CJ) were associated with relatively faster reaction times $\left(F_{(1,21)}=\right.$ $14.7 ; p=0.001$ for the main effect of WM maintenance) than control tasks (J and CJ), driven by a computation by WM interaction $\left(F_{(1,21)}=32.3 ; p<0.001\right)$ where the task with encoding, retrieval and numerical size judgment (E_RJ) had disproportionately fast reaction time, consistent with response preparation in this task (supplemental Fig. 2, available at www.jneurosci.org as supplemental material). Conversely, these WM tasks (E_RJ and E_CJ $)$ tended to have lower accuracy $\left(F_{(1,21)}=3.32 ; p=0.083\right.$ for the main effect of WM maintenance), driven by a computation by WM interaction, where the task engaging computation within WM (E_CJ) was performed with the lowest accuracy $\left(F_{(1,21)}=\right.$ 12.68; $p=0.002$ ) (supplemental Fig. 1, available at www. jneurosci.org as supplemental material).

There were no significant COMT-genotype group differences in age $\left(F_{(2,21)}=1.78 ; p>0.19\right)$, gender $\left(\chi_{(2)}^{2}=0.45 ; p>0.7\right)$, years of education $\left(F_{(2,21)}=0.094 ; p>0.9\right)$, and IQ $\left(F_{(2,21)}=0.8\right.$; $p>0.46)$. For accuracy and reaction time, there were no significant COMT differences across all the task response phases: numerical size judgment ( $\mathrm{J}$, accuracy, $F_{(2,21)}=0.37, p>0.69$; reaction time, $\left.F_{(2,21)}=0.65, p>0.5\right)$; computation and size judgment (CJ, accuracy, $F_{(2,21)}=1.05, p>0.37$; reaction time, $\left.F_{(2,21)}=0.73, p>0.4\right)$; encoding, retrieval and size judgment (E_RJ, accuracy, $F_{(2,21)}=0.35, p>0.7$; reaction time, $F_{(2,21)}=$ $0.29, p>0.7)$; encoding, computation in WM and size judgment (E_CJ, accuracy, $F_{(2,21)}=0.35, p>0.7$; reaction time, $F_{(2,21)}=$ $0.24, p>0.7)$. Thus, COMT genotype effects at the level of BOLD responses reflected information processing physiology and were not confounded by behavioral output.

\section{Task-related activation}

Among the control tasks, the motor task (M) predominantly engaged regions in the left premotor and motor cortices, caudate, and the bilateral parietal cortices (supplemental Table 1, available at www.jneurosci.org as supplemental material). Relative to this task, numerical size judgment (i.e., J > M contrast) entailed greater activation in regions in the bilateral VLPFC and superior parietal cortices. Numerical computation (i.e., CJ > J contrast) recruited further activation in the bilateral DLPFC, VLPFC, posterior parietal cortices, and striatum (Fig. 2, supplemental Table 1, available at www.jneurosci.org as supplemental material).

In the WM tasks (Fig. 2, supplemental Table 2, available at www.jneurosci.org as supplemental material), the encoding phase activated regions in the bilateral DLPFC, VLPFC, superior parietal lobules, and striatum. During retrieval (E_RJ $>M$ contrast), similar regions in the bilateral PFC, parietal cortices and striatum were activated. Relative to this, numerical computation in WM (i.e., E_CJ > E_RJ contrast) was associated with greater frontoparietal and striatal activation. Subtracting the effects of numerical computation to reflect the integration of information within WM across encoding and probe phases in the contrast E_CJ $>$ CJ resulted in bilateral DLPFC and posterior parietal activation (Fig. 2, supplemental Table 2, available at www. jneurosci.org as supplemental material).

\section{COMT Val > Met genotype effects in regions of interest}

The COMT Val>Met genotype effects on each cognitive task phase identified above were subsequently examined within functional ROIs anatomically located in the prefrontal cortex, posterior parietal cortex, and striatum that were significantly activated in the respective contrasts of interest (supplemental Tables 1, 2, bold type, available at www.jneurosci.org as supplemental material). There were no COMT genotype effects in the $\mathrm{M}$ task at the chosen thresholds. However, in numerical size judgment $(\mathrm{J}>$ $\mathrm{M}), C O M T \mathrm{Val}>\mathrm{Met}$ effects were observed in the right VLPFC ROI (BA44 peak 4214 12; $t=3.24 ; p<0.05$, corrected) (Fig. 2, supplemental Table 1, available at www.jneurosci.org as supplemental material). Activation related to additional numerical computation $(\mathrm{CJ}>\mathrm{J})$ correlated with COMT Val allele-load in the left DLPFC ROI (BA 46 peak -4242 20; $t=4.38$; $p<0.05$, corrected), right putamen (peak $18106 ; t=2.82 ; p<0.05$, corrected) and in bilateral inferior parietal lobules (BA 40, peak $-48-3458, t=4.64, p<0.02$, corrected; and $36-5456 ; t=$ $4.48 ; p<0.03$, corrected) (Fig. 2, supplemental Table 1, available at www.jneurosci.org as supplemental material).

During encoding (E) in the WM tasks, COMT Val $>$ Met genotype effect was observed in the right DLPFC ROI (BA 9, peak $345034 ; t=3.25 ; p<0.05$, corrected) (Fig. 2, supplemental 


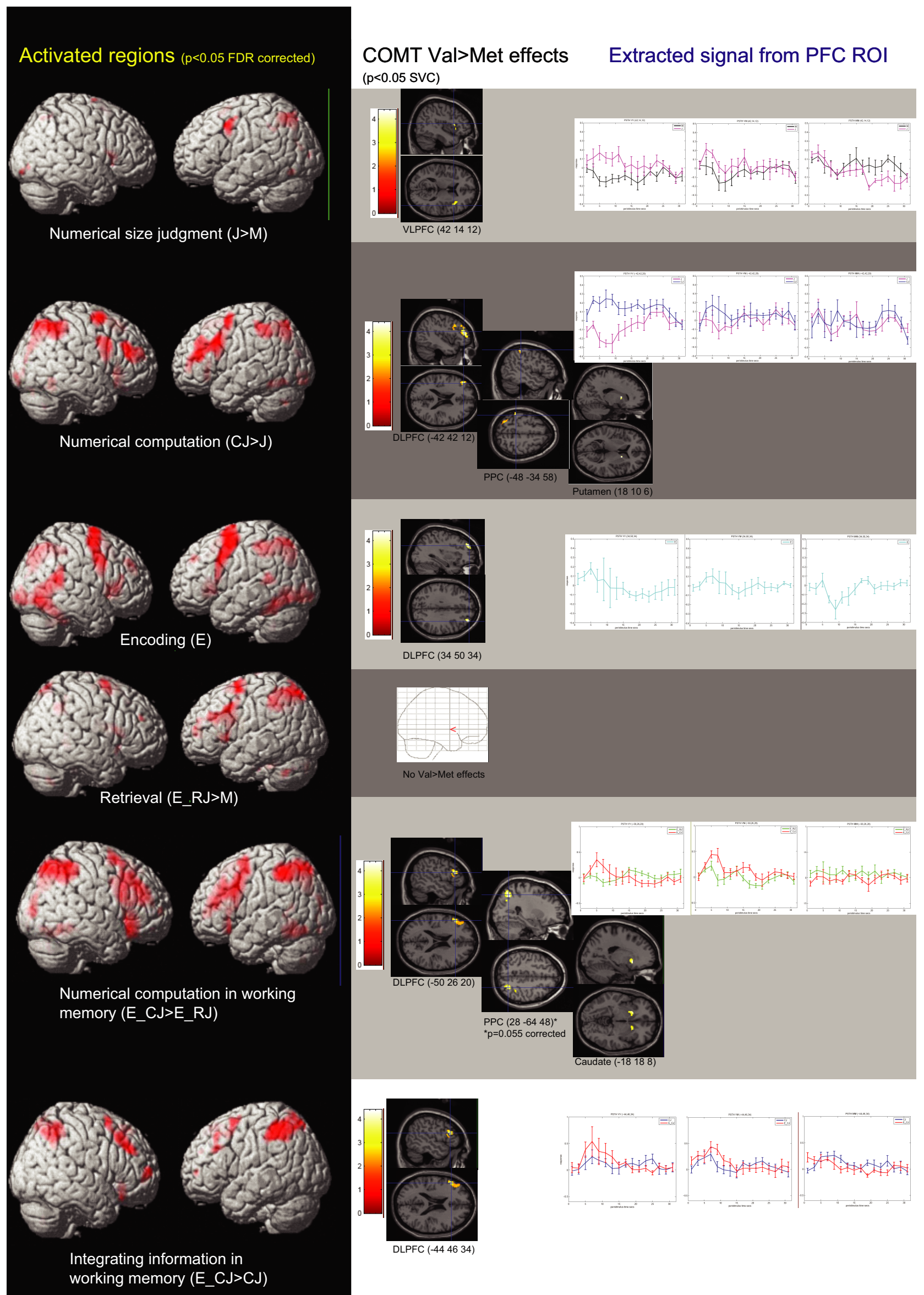

Figure 2. Regions activated in the contrasts of interest (left), corresponding ROls with COMT Val $>$ Met effects (middle), and extracted signal from the prefrontal ROl according to COMT genotype (right). 
Table 2, available at www.jneurosci.org as supplemental material). A weaker genotype effect was observed within ROIs in the left DLPFC (peak -2648 36; $t=2.92 ; p<0.004$, uncorrected), and right superior parietal lobule (peak $30-5052 ; t=3.12$; $p<$ 0.003 , uncorrected) but none in the striatal ROIs $(p>0.01$, uncorrected). At retrieval (E_RJ $>\mathrm{M})$, no COMT effects were observed in the parietal, frontal and striatal ROIs, even at $p<0.1$, uncorrected (Fig. 2, supplemental Table 2, available at www. jneurosci.org as supplemental material). To further examine encoding and retrieval processes, extracted signal from peak regions in the DLPFC with orthogonal main effects of the respective tasks (30 4036 in $\mathrm{E}>$ baseline and -36 3026 in E_RJ > M) (supplemental Table 2, available at www.jneurosci.org as supplemental material) showed a task-by-COMT interaction trend $\left(F_{(2,19)}=\right.$ $3.31 ; p=0.058$ ) (supplemental Figure 3, available at www. jneurosci.org as supplemental material) that would support the contention that COMT Val $>$ Met effects were observed at $\mathrm{E}>$ baseline but not at $\mathrm{E} \_\mathrm{RJ}>\mathrm{M}$ in activated DLPFC regions.

COMT Val allele-load also correlated with activation during numerical manipulation in WM (E_CJ $>$ E_RJ) at left DLPFC (peak $-502620 ; t=4.56 ; p<0.05$, corrected), right VLPFC (peak 5024 12; $t=4.97 ; p<0.05$, corrected), left caudate (peak $-1818-8 ; t=3.49 ; p<0.05$, corrected), and right inferior parietal lobule (BA 40, peak $28-6448 ; t=3.50 ; p=0.055$, corrected) (Fig. 2, supplemental Table 2, available at www. jneurosci.org as supplemental material). At the more lenient threshold, a region in the right caudate (peak 18160 ; $t=2.83$; $p<0.005$, uncorrected) and in the ROI at left superior parietal lobule (peak $-24-5860 ; t=3.10 ; p<0.003$, uncorrected) also showed COMT Val $>$ Met effects.

In the contrast examining integration of information in WM across encoding and probe phases (E_CJ $>$ CJ), COMTVal $>$ Met effects were observed in ROIs at the left DLPFC (peaks -444634 , $t=3.30, p<0.05$, corrected; $-522628, t=3.00, p<0.05$, corrected) (Fig. 2, supplemental Table 2, available at www. jneurosci.org as supplemental material). No COMT Val $>$ Met effects were observed at the caudate or posterior parietal cortex ( $p>0.1$, uncorrected). Thus, integrating information in WM was associated with greater dopaminergic modulation within this region of the DLPFC compared with calculation alone. This contention was supported by the observation that extracted parameter estimates from the respective tasks relative to numerical size judgment (i.e., E_CJ $>$ J and CJ $>$ J) also showed a task bygenotype interaction at this peak $\left(-444634 ; F_{(2,19)}=3.99 ; p<\right.$ $0.05)$. Here, the effect of COMT dopaminergic modulation was disproportionately greater in the task requiring further integration of information in WM than simple computation outside WM (Fig. 3). A higher-order interaction analysis of WM by computation by COMT, expressed as COMT Val $>$ Met effects in the contrast (E_CJ $>$ E_RJ $)>(C J>J)$, mapped to a similar DLPFC peak $(-403628 ; t=3.30 ; p=0.002$, uncorrected). Hence, these results converge on the possibility that manipulation within WM while subtracting out the effect of simple manipulation, or integrating information across time in WM, appears to engage much more dopaminergic effects at the DLPFC.

\section{WM network functional integration and COMT genotype}

Finally, we tested the prediction that the increased activation with COMT-Val allele load reflected poorer neural tuning and functional integration within the WM network (Spencer et al., 2004; Meyer-Lindenberg et al., 2005; Winterer et al., 2006). Functional coupling in the corticostriatal WM network during numerical computation in WM (E_CJ task phase) was examined in relation

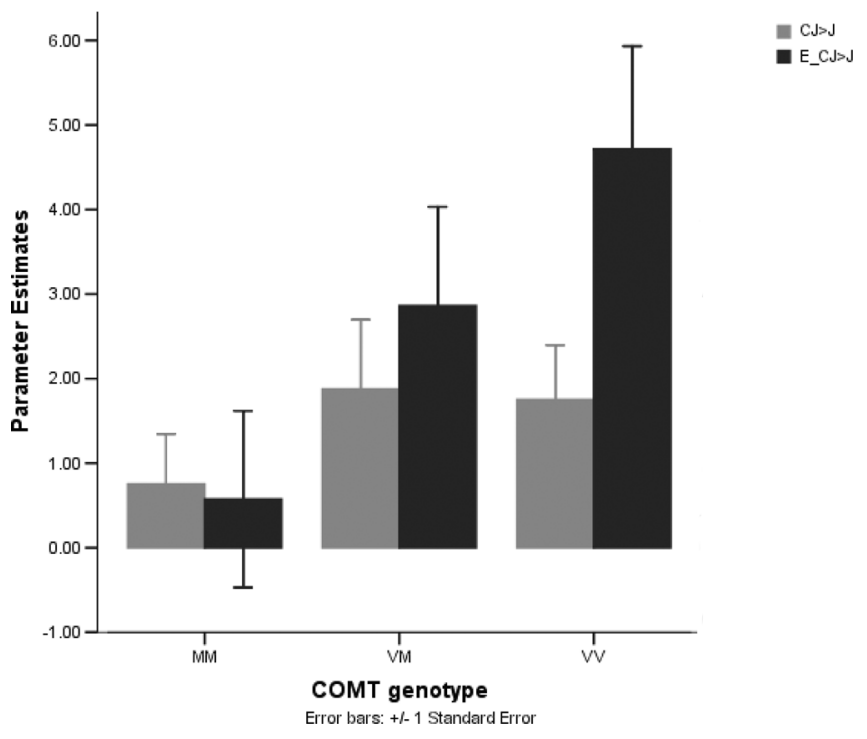

Figure 3. Relative to baseline numerical size judgment, extracted parameter estimates from the working memory manipulation task $\left(E_{-}(\mathrm{J}>\mathrm{J})\right.$ and numerical manipulation task $(\mathrm{CJ}>\mathrm{J})$ showed a task-by-genotype interaction at the DLPFC.

to COMT genotype using PPI (Friston et al. 1997). We found increased task-related functional integration with COMT-Met allele load, which putatively represented more optimal cortical DA function (Mattay et al., 2003; Meyer-Lindenberg et al., 2005). This occurred between the caudate seed ROI and regions in the DLPFC $(-363632 ; t=2.95 ; p=0.004)$ and parietal cortex (32-66 58, $t=3.22, p=0.002 ;-32-7446 ; t=2.54 ; p=0.01$ ). The opposite contrast relative to COMT-Val allele load yielded no prefrontal-parietal regions of increased coupling $(p>0.05)$. Thus, reduced WM task-related striatal-cortical functional coupling relative to cortical COMT-Val DA deficits (Chen et al., 2004) occurred in regions corresponding closely to those engaging increased COMT Val $>$ Met activation that was inefficient.

\section{Discussion}

Building on previous work implicating cortical DA indexed by COMT Val158Met genetic variation on prefrontal neural responses (Egan et al., 2001b; Gothelf et al., 2005; MeyerLindenberg et al., 2005, 2006; Tan et al., 2007), we found COMTrelated activation changes, putatively reflecting regions critically modulated by dopaminergic neural tuning and functional integration, that differentially mapped onto multiple levels of WM processing. There were no significant task performance differences across genotype, suggesting that the increased BOLD effects observed more directly reflected COMT Val-related neural signaling changes. This dopaminergic modulation occurred with a degree of spatial and process specificity over a network of hierarchical prefrontal, parietal and striatal regions. For numerical size comparison, COMT dopaminergic modulation was evident within the VLPFC. Numerical computations further engaged dopaminergic modulation in DLPFC, as well as in number-sensitive posterior parietal regions, and secondarily, the striatum (see below). Additional temporal integration of information within WM was associated with disproportionately increased dopaminergic effects only at the DLFPC. Dopaminergic modulation in anterior DLPFC was observed during WM encoding but, critically, not during its retrieval. These findings potentially integrate dopaminergic tuning of signal-to-noise in basic cortical assem- 
blies with their roles within human brain networks during the orchestration of information updating and stabilization in WM.

\section{Dopamine and hierarchical prefrontal working memory processes}

The findings of dopaminergic modulation by COMT at various levels of WM task complexity in prefrontal regions are consistent with suggestions that the DA system is implicated in mediating these hierarchical prefrontal cognitive control processes. In keeping with previous conceptualizations that the VLPFC is associated with simpler cognitive control processes relative to the DLPFC (Liddle et al., 2001; Koechlin et al., 2003), the baseline two-choice numerical size comparison task was associated with activation at the VLPFC. That this region also evidenced COMT $\mathrm{Val}>$ Met effects implicates dopaminergic modulation in this process, too.

When information was encoded and actively maintained "across time," associated dopaminergic modulation occurred in the DLPFC. This is consistent with the preeminent role attributed to DLPFC dopamine during WM (Goldman-Rakic 1996). Furthermore, we found a degree of process-specificity in relation to COMT dopaminergic modulation. In particular, although the DLPFC was activated in both the encoding and retrieval phases in WM, COMT dopaminergic modulation appeared more prominent in the former, but not in the latter task-phase. These results build on neural recordings in which dopaminergic tuning mechanisms were observed to play key roles in encoding and actively maintaining information signal, and in protecting or stabilizing them against neural noise (Williams and Goldman-Rakic, 1995; Seamans and Yang, 2004; Vijayraghavan et al., 2007). Human fMRI models have also predicted that the DLPFC might be implicated in active maintenance against distracters (Sakai et al., 2002). Our findings are consistent with the suggestion that earlier encoding and active maintenance processes engaged DAdependent mechanisms as they dynamically gate and stabilize new information in prefrontal neurons (Durstewitz et al., 2000; Seamans and Yang, 2004). Conversely, simple retrieval of thus stabilized or encoded information appeared to engage relatively less DA-dependent mechanisms.

If indeed dopaminergic modulation is implicated in gating and stabilization processes, executive functions involving manipulation and the rapid updating and stabilization of new information should also critically engage dopaminergic modulation at DLPFC. Our findings at numerical computation, during which COMT dopaminergic modulation occurred in the DLPFC (at BA46, -42 4220 during CJ > J and -52 2620 during E_CJ > E_RJ) were consistent with this hypothesis. The findings were also consistent with earlier work whereby prefrontal activation extended to the DLPFC during executive tasks (Callicott et al., 1999; D'Esposito et al., 1999; Koechlin et al., 2003; Tan et al., 2005, 2006), as well as engaged COMT dopaminergic modulation (Egan et al., 2001b; Mattay et al., 2003; Meyer-Lindenberg et al., 2005; Tan et al., 2007). Moreover, performing numerical computations in WM versus numerical computations alone (E_CJ > $\mathrm{CJ}$ ), in a contrast representing the temporal integration of probe with encoded information in WM, elicited greater activation as well as dopaminergic modulation in an anterior-dorsal PFC region (BA46, -44 46 34). This dissociation was more striking when these two tasks were considered relative to the baseline numerical comparison task $(\mathrm{J})$, where a statistical interaction at this locale suggests that combined temporally integrating information and performing arithmetic transformations disproportionately engaged greater activation magnitude and dopaminer- gic modulation than the latter process alone (Fig. 3). Thus, this anterior region in the DLPFC appeared critically engaged during DA-dependent processing of higher-order temporal or episodic aspects of WM (Sakai and Passingham, 2002; Koechlin et al., 2003), whereas DA-dependent processes in inferior-posterior $\mathrm{PFC}$ regions mediated the manipulation of information.

\section{Striatum and posterior cortex in arithmetic transformations}

To examine differential cortical dopaminergic modulation at other key nodes in the WM network, we explored COMT effects at the posterior parietal cortical and striatal ROIs. Given that COMT plays a relatively minor role in DA catabolism outside the cerebral cortex (Karoum et al., 1994; Gogos et al., 1998), striatal differences were likely to have been an indirect feedback effect mediated by changes in the PFC (Weinberger, 1987; Grace, 2000; Akil et al., 2003; Meyer-Lindenberg et al., 2005). In this context, we observed that prefrontal dopaminergic modulation of striatal activation was more prominent during numerical computation tasks. These effects were less apparent during encoding, retrieval, and in the contrast examining the temporal integration of information. The relatively specific engagement of prefrontal-parietal-striatal dopaminergic modulation during these computational tasks supports their role in the effective control of rapid switching and stabilization processes intrinsic in such tasks engaging the manipulation of information. This is also consistent with models predicting basal ganglia coupling of prefrontal cortex and modality-specific (e.g., numerical) regions in the posterior cortex, to effect this highly selective information transformation and updating; these models also propose that DA is critical in the implementation of these targeted gating processes (Gruber et al., 2006; O'Reilly and Frank, 2006).

However, processes involved in the manipulation of information might be distinguished from those engaged in the temporal integration of information in WM. The latter were associated with more prominent dopaminergic modulation within the anterior DLPFC rather than in the striatum or posterior parietal cortex. This observation argues that dopaminergic processes in these DLPFC regions might more critically mediate higher-order temporal processes, such as when contextual information is encoded for future operations, or when new probe information has to be integrated with that encoded previously. Together with propositions that these higher-order processes engage more overall inhibitory (Deco and Rolls, 2005) or biasing (Miller and Cohen, 2001) cognitive control that could engage greater dopamine $\mathrm{D}_{1}$ than $\mathrm{D}_{2}$ mechanisms (Durstewitz et al., 2000; Seamans and Yang, 2004), the former postulated to predominate in the PFC (Goldman-Rakic et al., 1990), one might speculate that our systems-level findings at these DLPFC regions could reflect greater $\mathrm{D}_{1}$ dopaminergic modulation during higher-order temporal integration of information. Conversely, rapid updating in manipulation involving the DLPFC, striatum and posterior cortex might reflect the engagement of predominantly $\mathrm{D}_{2}$ mechanisms (Goldman-Rakic, 1995; Mink, 1996; Seamans and Yang, 2004; Gruber et al., 2006; O’Reilly and Frank, 2006). Nevertheless, the basic neural and computational models have not incontrovertibly described the biology of $\mathrm{D}_{1}$ and $\mathrm{D}_{2}$ receptor mechanisms in prefrontal cortex, and differential BOLD activation is of limited inferential power to resolve controversies that remain. Accordingly, more direct receptor-imaging studies might be indicated in the future to elucidate how these mechanisms could indeed dissociate executive control functions within the WM network. 


\section{Limitations}

In this study, we have made the assumption that given the absence of performance differences across COMT genotype groups, the relatively increased BOLD activation observed as a function of COMT-Val allele load, also noted previously (Egan et al., 2001b; Mattay et al., 2003; Gothelf et al., 2005; Bertolino et al., 2006; Meyer-Lindenberg et al., 2006; Tan et al., 2007), corresponded spatially to brain regions wherein related dopaminergic modulation of neural signaling might be critical. However, although the finding of differential COMT activity in DLPFC were grounded in postmortem studies (Chen et al., 2004), the precise mechanism by which the increased BOLD activation correlates with neural DA signaling remains to be precisely determined. Nevertheless, our findings on prefrontal-parietal-striatal functional coupling suggest that this increased BOLD response corresponds to a less efficient and less functionally integrated network, augmenting the possibility that these effects reflect fundamental changes in DA-mediated tuning of signal-to-noise processing in cortical assemblies (Winterer and Weinberger, 2004) and compensatory processes.

\section{Conclusion}

We have used COMT genotype as a proxy of cortical DA signaling in healthy human volunteers to assay, with fMRI, differential dopaminergic modulation of neural circuitry involved in cognitive subcomponents of WM. Dopamine-dependent prefrontal cortical processes appear to critically mediate hierarchically dissociable executive control functions. Higher-order temporal operations to update and stabilize relevant new information, but less so the retrieval of already stabilized representations, engaged relatively specific DLPFC dopaminergic processes. Manipulating and rapidly updating representations involved dopaminergic modulation in a larger network of prefrontal, posterior cortical and striatal regions.

\section{References}

Akil M, Kolachana BS, Rothmond DA, Hyde TM, Weinberger DR, Kleinman JE (2003) Catechol-O-Methyltransferase genotype and dopamine regulation in the human brain. J Neurosci 23:2008-2013.

Alexander GE, DeLong MR, Strick PL (1986) Parallel organization of functionally segregated circuits linking basal ganglia and cortex. Annual Rev of Neuroscience 9:357-381.

Bertolino A, Caforio G, Blasi G, De Candia M, Latorre V, Petruzzella V, Altamura M, Nappi G, Papa S, Callicott JH, Mattay VS, Bellomo A, Scarabino T, Weinberger DR, Nardini M (2004) Interaction of COMT Val108/158 Met genotype and Olanzapine treatment on prefrontal cortical cunction in patients with schizophrenia. Am J Psychiatry 161:1798-1805.

Bertolino A, Blasi G, Latorre V, Rubino V, Rampino A, Sinibaldi L, Caforio G, Petruzzella V, Pizzuti A, Scarabino T, Nardini M, Weinberger DR, Dallapiccola B (2006) Additive effects of genetic variation in dopamine regulating genes on working memory cortical activity in human brain. J Neurosci 26:3918-3922.

Callicott JH, Mattay VS, Bertolino A, Callicott JH, Mattay VS, Bertolino A, Finn K, Coppola R, Frank JA, Goldberg TE, Weinberger DR (1999) Physiological characteristics of capacity constraints in working memory as revealed by functional MRI. Cereb Cortex 9:20-26.

Chen J, Lipska BK, Halim N, Ma QD, Matsumoto M, Melhem S, Kolachana BS, Hyde TM, Herman MM, Apud J, Egan MF, Kleinman JE, Weinberger DR (2004) Functional analysis of genetic variation in catechol-Omethyltransferase (COMT): effects on mRNA, protein, and enzyme activity in postmortem human brain. Am J Hum Genet 75:807-821.

Deco G, Rolls ET (2005) Attention, short-term memory, and action selection: a unifying theroy. Prog Neurobiol 76:236-256.

Dehaene S, Piazza M, Pinel P, Cohen L (2003) Three parietal circuits for number processing. Cogn Neuropsychol 20:487-506.

D’Esposito M, Postle BR, Ballard D, Lease J (1999) Maintenance versus ma- nipulation of information held in working memory: an event-related fMRI study. Brain Cogn 41:66-86.

Durstewitz D, Seamans JK, Sejnowski TJ (2000) Neurocomputational models of working memory. Nat Neurosci [Suppl] 3:1184-1191.

Egan MF, Goldberg TE, Gscheidle T, Weirich M, Rawlings R, Hyde TM, Bigelow L, Weinberger DR (2001a) Relative risk for cognitive impairments in siblings of patients with schizophrenia. Biol Psychiatry 50:98-107.

Egan MF, Goldberg TE, Kolachana BS, Callicott JH, Mazzanti CM, Straub RE, Goldman D, Weinberger DR (2001b) Effect of COMT Val108/158 Met genotype on frontal lobe function and risk for schizophrenia. Proc Natl Acad Sci USA 98:6917-6922.

Friston KJ, Buechel C, Fink GR, Morris J, Rolls E, Dolan RJ (1997) Psychophysiological and modulatory interactions in neuroimaging. NeuroImage 6:218.

Fuster JM (1997) The prefrontal cortex: anatomy, physiology, and neuropsychology of the frontal lobe. Philadelphia: Lippincott-Raven.

Genovese CR, Lazar NA, Nichols T (2002) Thresholding of statistical maps in functional imaging using the false discovery rate. NeuroImage $15: 870-878$.

Gogos JA, Morgan M, Luine V, Ogawa S, Pfaff D, Karayiourgou M (1998) Catechol-o-methyltransferase-deficient mice exhibit sexually dimorphic changes in catecholamine levels and behavior. Proc Natl Acad Sci USA 95:9991-9996.

Goldman-Rakic PS (1995) Cellular basis of working memory. Neuron 14:477.

Goldman-Rakic PS (1996) Regional and cellular fractionation of working memory. Proc Natl Acad Sci USA 93:13473-13480.

Goldman-Rakic PS, Lidow MS, Gallager DW (1990) Overlap of dopaminergic, adrenergic, and serotoninergic receptors and complementarity of their subtypes in primate prefrontal cortex. J Neurosci 10:2125-2138.

Gothelf D, Eliez S, Thompson T, Gothelf D, Eliez S, Thompson T, Hinard C, Penniman L, Feinstein C, Kwon H, Jin S, Jo B, Antonarakis SE, Morris MA, Reiss AL (2005) COMT genotype predicts longitudinal cognitive decline and psychosis in 22q11.2 deletion syndrome. Nat Neurosci $8: 1500-1502$.

Grace AA (2000) Gating of information flow within the limbic system and the pathophysiology of schizophrenia. Brain Res Brain Res Rev 31:330-341.

Gruber AJ, Dayan P, Gutkin BS, Solla SA (2006) Dopamine modulation in the basal ganglia locks the gate to working memory. J Comput Neurosci 20:153-166.

Hubbard EM, Piazza M, Pinel P, Dehaene S (2005) Interactions between number and space in parietal cortex. Nat Rev Neurosci 6:435-448.

Karoum F, Chrapusta SJ, Egan MJ (1994) 3-methoxytyramine is the major metabolite of released dopamine in the rat frontal cortex: reassessment of the effects of antipsychotics on the dynamics of dopamine release and metabolism in the frontal cortex, nucleus accumbens and striatum by a simple two-pool model. J Neurochem 63:972-979.

Koechlin E, Ody C, Kouneiher FT (2003) The architecture of cognitive control in the human prefrontal cortex. Science 302:1181-1185.

Liddle P, Kiehl K, Smith A (2001) Event-related fMRI study of response inhibition. Hum Brain Mapp 12:100-109.

Mattay VS, Goldberg TE, Fera F, Hariri AR, Tessitore A, Egan MF, Kolachana B, Callicott JH, Weinberger DR (2003) Catechol O-methyltransferase val158-met genotype and individual variation in the brain response to amphetamine. Proc Natl Acad Sci USA 100:6186-6191.

Meyer-Lindenberg A, Kohn PD, Kolachana B, Kippenhan S, McInerney-Leo A, Nussbaum R, Weinberger DR, Berman KF (2005) Midbrain dopamine and prefrontal function in humans: interaction and modulation by COMT genotype. Nat Neurosci 8:594-596.

Meyer-Lindenberg AS, Nichols T, Callicott JH, Ding J, Kolachana B, Buckholtz J, Mattay VS, Egan M, Weinberger DR (2006) Impact of complex genetic variation in COMT on human brain function. Mol Psychiatry $11: 867-877$.

Miller EK, Cohen JD (2001) An integrative theory of prefrontal cortex function. Ann Rev Neurosci 24:167-202.

Mink JW (1996) The basal ganglia: focused selection and inhibition of competing motor programs. Prog Neurobiol 50:381-425.

O’Reilly RC (2006) Biologically based computational models of high-level cognition. Science 314:91-94.

O'Reilly RC, Frank MJ (2006) Making working memory work: a computa- 
tional model of learning in the prefrontal cortex and basal ganglia. Neural Comput 18:283-328.

Oldfield R (1971) The assessment and analysis of handedness: the Edinburgh inventory. Neuropsychologia 9:97-113.

Sakai K, Passingham RE (2002) Prefrontal interactions reflect future task operations. Nat Neurosci 6:75-81.

Sakai K, Rowe JB, Passingham RE (2002) Active maintenance in prefrontal area 46 creates distractor-resistant memory. Nat Neurosci 5:479-484.

Sawaguchi T, Goldman-Rakic PS (1991) D1 dopamine receptors in prefrontal cortex: involvement in working memory. Science 251:947-950.

Seamans JK, Yang CR (2004) The principal features and mechanisms of dopamine modulation in the prefrontal cortex. Prog Neurobiol 74:1-57.

Seamans JK, Gorelova N, Durstewitz D, Yang CR (2001) Bidirectional dopamine modulation of GABAergic inhibition in prefrontal cortical pyramidal neurons. J Neurosci 21:3628.

Spencer KM, Nestor PG, Perlmutter R, Niznikiewicz MA, Klump MC, Frumin M, Shenton ME, McCarley RW (2004) Neural synchrony indexes disordered perception and cognition in schizophrenia. Proc Natl Acad Sci USA 101:17288-17293.

Tan HY, Choo WC, Fones CSL, Chee MWL (2005) fMRI study of maintenance and manipulation processes within working memory in firstepisode schizophrenia. Am J Psychiatry 162:1849-1858.

Tan HY, Sust S, Buckholtz JW, Mattay VS, Meyer-Lindenberg A, Egan MF, Weinberger DR, Callicott JH (2006) Dysfunctional prefrontal regional specialization and compensation in schizophrenia. Am J Psychiatry 163:1969-1977.

Tan HY, Chen Q, Sust S, Buckholtz JW, Meyers JD, Egan MF, Mattay VS, Meyer-Lindenberg A, Weinberger DR, Callicott JH (2007) Epistasis be- tween catechol-O-methyltransferase and type II metabotropic glutamate receptor 3 genes in working memory brain function. Proc Natl Acad Sci USA 104:12536-12541.

Tanaka SC, Doya K, Okada G, Ueda K, Okamoto Y, Yamawaki S (2004) Prediction of immediate and future rewards differentially recruits cortico-basal ganglia loops. Nat Neurosci 7:887-893.

Tunbridge EM, Harrison PJ, Weinberger DR (2006) Catechol-oMethyltransferase, cognition, and psychosis: Val158Met and beyond. Biol Psychiatry 60:141-151.

Vijayraghavan S, Wang M, Birnbaum SG, Williams GV, Arnsten AFT (2007) Inverted- $\mathrm{U}$ dopamine $\mathrm{D} 1$ receptor actions on prefrontal neurons engaged in working memory. Nat Neurosci 10:376-384.

Wager TD, Nichols TE (2003) Optimization of experimental design in fMRI: a general framework using a genetic algorithm. NeuroImage 18:293-309.

Weinberger DR (1987) Implications of normal brain development for the pathogenesis of schizophrenia. Arc Gen Psychiatry 44:660-669.

Williams GV, Goldman-Rakic PS (1995) Modulation of memory fields by dopamine D1 receptors in prefrontal cortex. Nature 376:572-575.

Winterer G, Weinberger DR (2004) Genes, dopamine and cortical signalto-noise ratio in schizophrenia. Trends Neurosci 27:683-690.

Winterer G, Egan MF, Kolachana BS, Goldberg TE, Coppola R, Weinberger DR (2006) Prefrontal electrophysiologic "noise" and catechol-Omethyltransferase genotype in schizophrenia. Biol Psychiatry 60:578-584.

Worsley KJ, Marrett S, Neelin P, Vandal AC, Friston KJ, Evans AC (1996) A unified statistical approach for determining significant signals in images of cerebral activation. Hum Brain Mapp 4:58-73. 